\title{
Questes
}

Revue pluridisciplinaire d'études médiévales

\section{"Le Coup de Melun » : trahison, châtiment et erreurs de datation dans diverses sources narratives (fin $\mathrm{X}^{\mathrm{e}}-\mathrm{XI}^{\mathrm{e}}$ siècle)}

Damien Varenne

\section{CpenEdition}

\section{Journals}

Édition électronique

URL : http://journals.openedition.org/questes/4224

DOI : $10.4000 /$ questes.4224

ISSN : 2109-9472

\section{Éditeur}

Les Amis de Questes

\section{Édition imprimée}

Date de publication : 30 octobre 2015

Pagination : 33-47

ISSN : 2102-7188

\section{Référence électronique}

Damien Varenne, « «Le Coup de Melun » : trahison, châtiment et erreurs de datation dans diverses sources narratives (fin $x^{e}-x_{1}^{e}$ siècle) », Questes [En ligne], 30 | 2015, mis en ligne le 01 novembre 2015 consulté le 01 mai 2019. URL : http://journals.openedition.org/questes/4224 ; DOI : 10.4000/ questes. 4224 


\title{
« Le Coup de Melun » : trahison, châtiment et erreurs de datation dans diverses sources narratives (fin $\mathrm{X}^{\mathrm{e}}-\mathrm{XI}^{\mathrm{e}}$ siècle)
}

\author{
Damien VARENNE \\ Université Paris-Sorbonne
}

Le règne d'Hugues Capet, roi des Francs, n'est pas l'un des plus documentés qui soient. Cependant, cette période bénéficie de l'éclairage, certes incomplet, que lui apporte le récit longtemps décrié de Richer de Reims. S'il ne peut prétendre apporter le récit circonstancié et exhaustif des événements de la période 987-996, il permet d'avoir une connaissance assez précise de quelques-unes des péripéties les plus marquantes de ces années.

Parmi celles-ci, celle que Dominique Barthélémy a appelée le « coup de Melun ${ }^{1}{ }^{n}$ 'est pas la plus connue, mais certainement l'une des plus étranges et intéressantes. Ce récit de la prise de Melun par Eudes I ${ }^{\mathrm{er}}$ de Blois en 991 grâce à la trahison de son châtelain, puis de sa reconquête par l'armée royale, suivie du châtiment exemplaire dudit châtelain, prend une valeur morale sur laquelle Richer insiste tout particulièrement, en même temps qu'il nous apporte de précieuses informations sur les mentalités du temps.

Cet événement a aussi suscité des débats chez les historiens quant à sa datation, suite à une erreur commise par l'un des chroniqueurs qui a rapporté les faits. Cette erreur, nous le verrons, n'est pas anodine et nous

\footnotetext{
${ }^{1}$ Dominique Barthélémy, La Société dans le comté de Vendôme de l'an Mil au XIV siècle, Fayard, 1993, p. 282 et 318.
} 
apporte quelques renseignements sur la manière dont étaient écrites les sources narratives du temps.

L'objet de cette étude est donc double :

- réfléchir sur ce que les nombreuses erreurs de datations des sources narratives des $\mathrm{X}^{\mathrm{e}}-\mathrm{XI}^{\mathrm{e}}$ siècles, au-delà des problèmes qu'elles peuvent nous poser sur le plan strictement technique de la chronologie, peuvent nous enseigner quand on prend la peine de s'interroger sur ce qui a pu les susciter.

- analyser la conception qu'ont les chroniqueurs (et au-delà les hommes) de ces siècles de la trahison, des traitres et des corrupteurs, et des châtiments qu'ils encourent.

\section{Les sources}

Les sources qui se rapportent à la prise de Melun sont presque exclusivement narratives. La seule exception est un diplôme d'Hugues Capet, sur lequel nous reviendrons, car il apporte un élément d'importance pour la datation de l'événement.

L'ouvrage les Quatre livres d'histoire de Richer de Reims est la première source, la plus ancienne et la plus importante. Le moine de Saint-Rémi développe le récit de la prise de la ville et de sa reprise par l'armée royale, agrémentant le tout de dialogues assez savoureux, bien que probablement inventés ${ }^{2}$. Il s'agit de la version de l'événement la plus développée et la plus riche en enseignements pour notre étude.

On trouve une autre version de l'événement, beaucoup plus succincte, dans l'Historia Francorum Senonensis ${ }^{3}$, petite chronique

\footnotetext{
${ }^{2}$ Richer de Reims, Histoire de France, éd. Robert Latouche, Paris, Les Belles Lettres, coll. «Les Classiques de l'histoire de France au Moyen Âge», 2 vol., vol. II, p. 267-275.

${ }^{3}$ Historia Francorum Senonensis, dans Monumenta Germaniae Historica, Scriptores, 9, p. 364-369.
} 
d'origine sénonaise (comme le suggère son nom), probablement écrite peu après 1015.

De nombreuses autres sources rapportant la prise de Melun ne font que reprendre, parfois mot pour mot, l'Historia Francorum Senonensis. Parmi ces nombreux écrits, citons la Chronique d'Hugues de Fleury ${ }^{4}$, la Chronica Regum Francorum ${ }^{5}$ écrite à l'abbaye de Saint-Denis au XII ${ }^{e}$ siècle, La Chronique de Saint-Pierre-le-Vif, écrite dans la première moitié du XII ${ }^{\mathrm{e}}$ siècle et longtemps attribuée au moine Clarius ${ }^{6}$, ainsi que d'autres chroniques écrites en latin et en ancien français, émanant souvent de l'abbaye de Saint-Denis ${ }^{7}$.

Eudes de Saint-Maur, dans sa Vita Burchardi ${ }^{8}$, donne sa propre version des événements qui introduisent le récit de la rivalité opposant Bouchard à Eudes de Blois, insistant beaucoup plus que les autres, bien sûr, sur le personnage de Bouchard le Vénérable. Notons que cette source, pas plus que Richer, ne date le siège.

Guillaume de Jumièges ${ }^{9}$, suivi par Orderic Vital, donne sa propre version du siège de Melun, en insistant particulièrement sur le rôle qu'y a joué le duc de Normandie et en livrant une foule de détails sur la bataille,

${ }^{4}$ Hugues de Fleury, Chronique, dans le Recueil des historiens des Gaules et de la France, éd. Martin Bouquet et Léopold Delisle, Paris, Palmé, 1869-1880, 19 vol., t. X, p. 220. Le numéro de page est celui où l'on trouve le passage se référant à la prise de Melun. Le Recueil des historiens des Gaules et de la France sera désormais abrégé en RHF.

${ }^{5}$ Ibid., p. 302.

${ }^{6}$ Chronique de Saint-Pierre-le-Vif de Sens, dite de Clarius, éd. Robert-Henri Bautier et Monique Gilles, Paris, Édition du Centre National de la Recherche Scientifique, coll. «Sources d'histoire médiévale », 1979, p. 104.

${ }^{7}$ Toutes sont présentes dans les RHF.

${ }^{8}$ Eudes de Saint-Maur, Vie de Bouchard le Vénérable, comte de Vendôme, de Corbeil, de Melun et de Paris, $X^{e}$ et XI siècles, éd. Charles Bourel de la Roncière, Paris, Picard, coll. "Collection de textes pour servir à l'étude et à l'enseignement de l'histoire $», 1892$, p. 18-19.

${ }^{9}$ Guillaume de Jumièges, Gesta Normannorum ducum, éd. Jean Marx, Paris, Picard, 1914. 
qui viennent corroborer la version qui en est donnée par Richer, s'ils ne s'inspirent pas directement de cette source.

Mentionnons enfin un passage du De Majoratu et Senescalcia Franciae $^{10}$, source angevine, qui, bien que comportant de nombreuses erreurs de chronologie ${ }^{11}$, pourrait évoquer le rôle joué par le comte Foulques Nerra dans les événements qui nous occupent.

Il ne faut pas oublier non plus la seule source non narrative de notre corpus, qui, si elle ne fait pas directement référence aux événements que nous allons étudier, en est la conséquence. Il s'agit d'un diplôme d'Hugues Capet et de Robert le Pieux daté du 16 septembre $991^{12}$ qui donne les monastères Saint-Père et Saint-Etienne de Melun à l'archevêque de Sens pour que celui-ci procède à leur restauration, suite à ce qui semble être, sans que cela soit explicite, les destructions consécutives à la reprise de la ville. Ce diplôme est important car il permet de confirmer que les événements de Melun se sont déroulés en $991^{13}$.

${ }^{10}$ Chroniques d'Anjou, éd. Paul Marchegay et André Salmon, Paris, Renouard, coll. «Publications pour la Société de l'histoire de France », 1856.

${ }^{11}$ Le récit met en effet aux prises Geoffroy Grisegonelle et Thibaut de Champagne et non Foulques Nerra et Eudes de Blois comme cela eût dû être le cas.

${ }^{12}$ Il est édité dans les RHF, op. cit., t. X, p. 559-560.

${ }^{13}$ Ferdinand Lot rattache cet acte royal à la prise de Melun et en fait un de ses arguments pour confirmer la date de 991. Voir Ferdinand Lot, «La Date de naissance du roi Robert II et le siège de Melun », dans Mélanges Julien Havet, dir. Charles Nerlinger, Paris, Leroux, 1895, p. 149-156. Pour d'autres arguments du même auteur appuyant la date de 991, voir Ferdinand Lot, Études sur le règne d'Hugues Capet et la fin $d u X^{e}$ siècle, Paris, Bouillon, coll. «Bibliothèque de l'École des hautes études. Sciences historiques et philologiques », 1903, p. 159162. 


\section{Les faits}

Que s'est-il donc passé à Melun pour que tant de chroniqueurs rapportent les faits, parfois de manière fort fouillée ? ${ }^{14}$

Si l'on en croit les auteurs médiévaux, la prise de la ville par Eudes $\mathrm{I}^{\text {er }}$ de Blois serait due à des motifs stratégiques et moraux à la fois. D'abord, nous dit Richer, Eudes cherche un port fluvial sur la Seine où faire passer ses troupes. Melun correspond de manière idéale à ce qu'il cherche et viendrait alors compléter les positions qu'il occupe déjà sur la Loire. À ce motif, somme toute assez prosaïque, Eudes de Saint-Maur ajoute la jalousie qui ronge Eudes vis-à-vis de celui qui est alors comte de Melun, mais aussi de Paris, Vendôme et Corbeil, Bouchard le Vénérable, proche entre tous du roi Hugues Capet.

Eudes s'emploie alors à suborner le vassal de Bouchard affecté à la garde de la ville. Peut-être cet homme, appelé Gautier, était-il vicomte, ou bien simple châtelain ${ }^{15}$. Peu importe en fait, car nos sources ne le nomment plus par la suite que proditor, « le traitre ».

Vaincu par les arguments d'Eudes et, plus encore, par les promesses de récompenses dont celui-ci n'est pas avare, Gautier livre la place au comte de Blois, ce qui est un indice de la faiblesse relative du comte Bouchard dans les places qu'il ne pouvait contrôler directement ${ }^{16}$. Après un simulacre destiné à laisser l'illusion de la fidélité du vicomte à

${ }^{14}$ La totalité du récit est tirée avant tout de celui de Richer, avec quelques compléments venus de Guillaume de Jumièges et d'Eudes de Saint-Maur. Nous n'alourdirons donc pas le texte par de trop nombreuses notes de bas de pages qui ne feraient que renvoyer à ces œuvres dont les références sont déjà citées plus haut.

${ }^{15}$ Richer en fait un praeses, mot que Robert Latouche traduit par châtelain. L'Historia Francorum Senonensis en fait un miles. Le terme employé par Richer, lié à l'expression vir consularis, employée par le même auteur pour donner le rang de notre homme, laisse supposer qu'il était vicomte.

16 Dominique Barthélémy, La Société dans le comté de Vendôme de l'an Mil au XIV siècle, op. cit., p. 282. 
son seigneur précédent, Gautier est bien vite libéré et se voit confier par son nouveau seigneur la garde et la défense de la ville.

L'armée royale est en effet assemblée, composée, en plus du contingent royal, de celui du duc de Normandie Richard I ${ }^{\text {er }}$ et peut-être du comte d'Anjou Foulques Nerra, comme le suggère le récit passablement déformé du De Majoratu et Senescalcia Franciae ${ }^{17}$.

Après un siège dont nous ne donnerons pas les détails amplement fournis par Richer, la place est reprise. Le reste de la garnison et le châtelain sont faits prisonniers. La garnison est pardonnée mais Gautier ne bénéficie pas, lui, d'un tel traitement de faveur puisqu'il est pendu. Chose beaucoup plus inhabituelle (Richer nous dit «inusitate ludibrii genere ») sa femme l'est aussi, d'une manière particulièrement infâmante, puisqu'elle est pendue par les pieds et que, ses vêtements lui retombant alors de part et d'autre du corps (on finit d'ailleurs par les lui arracher), ses pudenda sont dévoilées.

Eudes, qui était, quant à lui, resté prudemment à l'écart de la ville avec le gros de ses troupes, ne peut que repartir, non sans soupirer sur la perte de sa garnison.

\section{À traître, traître et demi}

L'une des raisons pour lesquelles les événements de Melun ont visiblement frappé les chroniqueurs contemporains et leurs successeurs est le châtiment particulièrement infamant infligé à Gautier et à son épouse.

L'intérêt pour nous est que Richer livre au fil de sa narration une réflexion bien développée sur la faute morale que constitue la trahison, le

\footnotetext{
${ }^{17}$ Ferdinand Lot, Études sur le règne d'Hugues Capet et la fin du $X^{e}$ siècle, op. cit., p. 159-162.
} 
châtiment qu'elle mérite (ou non) et la place que prend au milieu de tout cela la figure du corrupteur.

Si presque toutes nos sources narratives évoquent le châtiment infligé au traître, seul Richer nous livre un récit détaillé, d'ailleurs sans doute largement imaginé, de la trahison elle-même. Que ce récit soit surtout né de l'imagination de Richer, qui n'a pas été témoin oculaire de l'événement, nous importe peu puisqu'il constitue malgré tout un témoignage important des mentalités du temps, d'autant plus que Richer a peut-être entendu raconter les faits de la bouche même d'Eudes ou de certains de ses proches lors d'un séjour de plusieurs semaines à Chartres $^{18}$.

Si l'on étudie en détail le récit de Richer, l'on se rend compte qu'il met en scène une série de plusieurs fautes.

La première est présentée du point de vue d'Eudes. Celui-ci en effet tempête : le comté de Melun n'est pas aux mains de son dépositaire légitime. En effet Eudes devrait disposer de cet honor pour deux raisons : d'abord parce qu'il est le plus puissant vassal du roi et ensuite parce que ses ancêtres l'ont possédé ${ }^{19}$. Cette faute-ci est la faute du roi «qui vit sans

${ }^{18}$ Richer raconte en effet au chapitre L de son livre un voyage qu'il fit à Chartres en 991 à la demande du clerc Héribrand, afin d'étudier les manuscrits d'Hippocrate. C'est peut-être auprès d'Héribrand et d'autres clercs que Richer puise les sources de son récit de la prise de Melun comme le suppose Robert Latouche dans Richer de Reims, op. cit., p. 267, note 2. C'est en tous cas l'hypothèse que fait Ferdinand Lot dans ses Études sur le règne d'Hugues Capet et la fin du $X^{e}$ siècle, op. cit., p. 158.

19 Henri d'Arbois de Jubainville a tenté de déterminer qui pouvait être l'ancêtre d'Eudes qui avait possédé Melun dans «L'Historien Richer et le siège de Melun en 999 », Bibliothèque de l'École des Chartes, vol. 20, 1859, p. 393-398. Après un raisonnement alambiqué, il déduit qu'il doit s'agir de Thibaud le Tricheur qui l'aurait reçu de son beau-père Herbert de Vermandois. Cependant, selon Michel Bur dans La Formation du comté de Champagne, v. 950-v. 1150, Nancy, Université de Nancy II, coll. «Annales de l'Est. Mémoire », 1977, p. 97, si Herbert de Vermandois a bien possédé Melun, rien ne prouve qu'il ait transmis cette ville à son beau-fils. Au contraire, Michel Bur postule que Melun a été récupérée par Hugues le Grand à la mort d'Herbert. 
gloire $^{20} \gg$ et favorise donc de moins puissants qu'Eudes, comme Bouchard. On peut noter au passage que chez Eudes de Saint-Maur la faute est inversée. C'est Eudes qui jalouse alors la puissance et la faveur royale dont dispose Bouchard. Le roi a ici favorisé la bonne personne ${ }^{21}$.

La deuxième faute, qui est la plus abondamment décrite dans le texte de Richer, est provoquée par la cupidité du châtelain. Celui-ci, après avoir abondé en faveur du bon droit d'Eudes à s'emparer de Melun, finit par révéler sa vraie nature en cédant aux promesses financières de ce dernier. Il fait alors office de nouveau Judas ${ }^{22}$ qui vend son maître (ou en l'occurrence le bien de celui-ci) pour ses trente deniers et la vague promesse d'une élévation dans l'ordre de la noblesse.

Troisième faute, enfin, commise celle-ci par Eudes : la trahison du serment prêté à Gautier à la fois par l'intermédiaire de son ambassadeur et de façon plus directe. "Si tu te livres à Eudes, affirme l'émissaire d'Eudes, que la responsabilité que tu redoutes retombe sur moi ». Or Eudes ne respecte pas le serment de son envoyé puisqu'il se défausse et abandonne le châtelain à son sort. Pire, il renie même l'accord avec ce dernier puisqu'il n'hésite pas à l'accabler de son mépris.

Cette énumération ne peut manquer de nous amener à nous interroger sur la valeur du serment dans la société de l'époque. De nombreux serments sont en effet trahis tout au long du récit: celui de Gautier bien sûr, mais aussi ceux de l'émissaire du comte de Blois et d'Eudes lui-même.

${ }^{20}$ Richer de Reims, Histoire de France, op. cit., p. 270.

${ }^{21}$ Eudes de Saint-Maur, Vie de Bouchard le Vénérable, op. cit., p. 18-19.

${ }^{22}$ On ne peut que mettre ici en parallèle le récit de la trahison de Gautier avec celle d'Adalbéron, racontée auparavant dans son ouvrage par Richer. Ce récit, d'après Robert Latouche, devait d'ailleurs à l'origine se placer juste avant celui de la prise de Melun renforçant le parallèle (voir Richer de Reims, Histoire de France, op. cit., p. 266, note 1). Adalbéron, au cours du repas précédant sa trahison émet force serments à Charles de Basse-Lorraine et se donne à lui-même le nom de Judas s'il les trahissait. 
Cependant il en est un dont on peut au contraire relever la force extrême, c'est celui de la garnison de Melun envers son châtelain (ou vicomte). Richer emploie même, pour évoquer leur comportement, le terme très fort de virtus. Ce serment passe même au-dessus de la « majesté royale », ce que reconnaît le roi en pardonnant à ces hommes, non sans leur avoir fait prêter hommage une nouvelle fois ${ }^{23}$.

Le roi joue d'ailleurs, par sa réaction, un rôle d'ordonnateur des valeurs régissant la société. En condamnant le traître Gautier et en pardonnant ${ }^{24}$ à la garnison, il montre où se trouve le bien, même si Eudes échappe dans l'immédiat à sa justice. De plus, le serment prêté par la garnison après sa libération l'est directement à sa personne. Ce faisant, il annule la contradiction qui faisait s'opposer pour ces hommes la fidélité à leur seigneur et celle au roi, contradiction bien réelle même en ces temps de faiblesse du pouvoir royal.

\section{Le châtiment du traître}

Nulle figure n'est plus maltraitée et méprisée que celle du traître Gautier dans l'ouvrage de Richer. Celui-ci prend bien soin, en effet, de ne plus le nommer par son nom dès lors qu'il a tourné casaque en faveur d'Eudes de Blois, ne l'affublant dès lors que par le sobriquet de proditor, «le traître », comme si sa faute lui avait fait perdre tout droit à une identité bien définie dans le récit.

Le roi n'est d'ailleurs pas en reste, en infligeant à Gautier et à son épouse un châtiment effroyable qui frappe les contemporains par son exemplarité et son aspect infamant, alors même que l'époque est plutôt à

\footnotetext{
${ }^{23}$ Richer de Reims, Histoire de France, op. cit., p. 272.

${ }^{24}$ Le pardon royal des fautes est par ailleurs chose assez classique au Moyen Âge comme le relève Ghislain Brunel dans «La Justice du roi de France vers l'an Mil », dans La Justice en l'an Mil, Paris, La Documentation française, coll. «Histoire de la justice », 2003.
} 
la modération entre gens de la noblesse, signe que la faute de Gautier le marque à jamais d'un signe d'infamie et le transforme en cible idéale du mépris universel.

Eudes lui-même, pourtant corrupteur et principal bénéficiaire de la trahison de Gautier, ne manque pas de l'accabler de son mépris lorsqu'il affirme être plus peiné par la perte de la garnison de Melun que par la «pendaison d'un traitre ${ }^{25}$ ».

Gautier est pendu, châtiment dont la forme n'est pas innocente puisqu'elle le rapproche de la figure de Judas Iscariote, le traître par excellence, dont il partage le sort final ${ }^{26}$.

Rien dans la mise en scène de la mort du châtelain n'est innocent. Le lieu choisi pour l'exécution est hautement symbolique : Richer affirme qu'il fut pendu près de la porte de la ville, sans doute sur le gibet public $^{27}$; voilà qui symbolise d'autant plus la déchéance de Gautier que celui-ci était le gardien, en tant que vicomte, de cette porte et le dépositaire de l'autorité publique.

Il est d'ailleurs intéressant de noter que cette exécution est tout entière marquée par la notion de vengeance : on punit Gautier par là où il a péché. Dépositaire de l'autorité publique il est châtié selon les formes publiques les plus humiliantes. Guillaume de Jumièges n'hésite d'ailleurs pas à employer l'expression de «talion », qui souligne d'autant plus que l'on rend à Gautier la monnaie de sa pièce ${ }^{28}$.

${ }^{25}$ Richer de Reims, Histoire de France, op. cit., p. 274.

${ }^{26}$ On retrouve ici le parallèle avec Adalbéron de Laon qui échappe cependant au châtiment, signe qu'il vaut mieux trahir pour le roi que contre celui-ci.

${ }^{27}$ Eudes de Saint-Maur, dans la Vie de Bouchard le Vénérable, op. cit., p. 19, place le lieu de l'exécution sur une colline, accentuant encore son aspect exemplaire.

${ }^{28}$ Notons au passage que, selon le dictionnaire de Jan Frederik Niermeyer et Co van de Kieft, Mediae Latinitatis lexicon minus, Leiden/Boston, Brill, 2002, le mot talio peut se traduire en allemand par Wergild, c'est à dire le prix du sang, ce qui nous ramène à la notion de faïde, si prégnante à l'époque mérovingienne. Nous avons donc bien affaire ici à une vengeance publique. 
Mais ce qui a le plus frappé les contemporains de l'événement c'est le châtiment de la femme de Gautier, pendue avec son mari. En effet, pendre une femme à cette époque est tout à fait exceptionnel, pour des raisons de pudeur élémentaire ${ }^{29}$, d'autant plus une femme noble qui doit de ce fait bénéficier d'une indulgence toute particulière.

De ce fait, le caractère exceptionnel de cette exécution vient encore renforcer l'impression de vengeance qui se dégageait déjà du sort réservé au mari. On veut faire de cette mise à mort un châtiment qui frappera et terrorisera longtemps les contemporains, ce qui a d'ailleurs manifestement réussi.

Le traitement de la femme de Gautier apparaît d'ailleurs si incroyable aux chroniqueurs médiévaux que certains tentent de l'expliquer par une participation de la femme à la décision de son mari. Ainsi de l'Historia Francorum Senonensis, qui n'hésite pas à associer la femme de Gautier à sa décision de livrer à Eudes la ville de Melun ${ }^{30}$.

Dans le récit de Richer l'ambiguïté du comportement d'Eudes de Blois est remarquable. Celui-ci, alors même qu'il est l'initiateur de la trahison de Gautier, est pardonné peu après par le roi au nom même des prétextes qu'il a donnés dès l'abord à sa trahison ${ }^{31}$. On ne peut d'ailleurs s'empêcher de mettre en parallèle le rôle joué par le comte de Blois avec celui du roi dans l'affaire de la trahison d'Adalbéron, racontée peu auparavant par Richer. Visiblement il est beaucoup plus facile d'être un puissant corrupteur qu'un faible corrompu dans la société de l'an Mil.

${ }^{29}$ Voir Valérie Toureille, Crime et châtiment au Moyen Âge. $V^{e}-X V^{e}$ siècle, Paris, Seuil, coll. «L'Univers historique», 2013, p. 256 et Nicole Gonthier, Le Châtiment du crime au Moyen Âge, Rennes, PUR, coll. « Histoire », 1998, p. 148149. Toutes deux soulignent la rareté de la pendaison des femmes au Moyen Âge tout en citant quelques cas, tous datés de la fin de la période. Il n'existe d'ailleurs que peu d'études sur ce sujet concernant le Haut Moyen Âge.

${ }^{30}$ Historia Francorum Senonensis, op. cit., p. 369 : «tradidit Walterius miles et uxor eius castellum Milidunum Odoni militi ».

${ }^{31}$ Richer de Reims, Histoire de France, op. cit., p. 278. 


\section{Quand l'erreur se fait féconde}

La plupart des sources et des historiens ayant travaillé sur l'affaire de Melun la datent de l'année 999. Il est pourtant assuré que les événements se situent en 991 , et ce depuis plus d'un siècle ${ }^{32}$. Pourquoi cette erreur?

Elle trouve son origine très tôt dans nos sources puisque l'Historia Francorum Senonensis, déjà, mentionne la prise et reprise de Melun à cette date. Comme, à l'exception de l'ouvrage de Richer, aucun texte plus ancien ne nous évoque cet épisode et que la plupart des ouvrages le mentionnant postérieurement, tout en présentant la même erreur, sont manifestement inspirés de notre source sénonaise, on peut facilement penser que c'est celle-ci qui est à l'origine de cette erreur.

Une analyse comparée du texte peut nous permettre de formuler une hypothèse sur l'origine de l'erreur de l'Historia Francorum Senonensis. Nous pouvons remarquer tout d'abord que, dans cette source, le récit de la trahison de Gautier se place immédiatement après la mention de la restauration de l'église Saint-Père de Melun par l'archevêque Seguin, elle aussi placée en 999.

\footnotetext{
${ }^{32}$ Henri d'Arbois de Jubainville, «L'Historien Richer et le siège de Melun en 999 », art. cit., p. 393-398 place ainsi l'événement en 999 et fait du comte de Blois Eudes II et non son père Eudes $\mathrm{I}^{\mathrm{er}}$. Il s'appuie sur l'analyse du discours que l'ambassadeur d'Eudes tient à Gautier qui ferait, selon lui, d'Eudes un jeune homme sans grandes ressources. Cette argumentation apparaît cependant bien fragile puisque basée sur des paroles sans doute inventées par Richer. Christian Pfister, dans les Études sur le règne de Robert le Pieux, Paris, Vieweg, coll. «Bibliothèque de l'École des hautes études. Sciences philologiques et historiques », 1885, p. 229, note 6, perpétue cette erreur. C'est Ferdinand Lot dans «La Date de naissance du roi Robert II et le siège de Melun », art. cit., qui rétablit la vérité en prouvant notamment que Richer n'a pas pu rédiger sa chronique après 997 et en rattachant le récit à un acte royal (cité plus haut dans nos sources) daté de 991.
} 
Si l'on rapproche l'Historia Francorum Senonensis d'une source sénonaise un peu postérieure, la Chronique d'Odorannus ${ }^{33}$, on remarque alors que, si cette dernière ne mentionne pas la trahison de Gautier, elle évoque bien la réfection de Saint-Père de Melun par Seguin et ce presque dans les mêmes termes ${ }^{34}$. Cependant cet évergétisme de l'archevêque n'est pas daté dans l'ouvrage d'Odorannus puisqu'il est mentionné dans une liste des bienfaits de Seguin qui vient couronner la mention de sa mort... en 999.

Les deux textes paraissent donc parents et cette parenté peut nous orienter dans le décryptage du premier. On peut en effet conclure du texte $^{35}$ que l'auteur anonyme de l'Historia Francorum Senonensis place l'affaire de Melun en 999 parce qu'il l'a reliée avec la réfection de l'abbaye située dans la même ville. Or il a situé cette dernière à cette date parce que dans la source qu'il a utilisée elle était vraisemblablement située, comme dans la Chronique d'Odorannus, au sein d'une longue liste des bienfaits de l'archevêque, écrite à l'occasion de sa mort, comme une rubrique nécrologique.

La proximité des mots utilisés par notre auteur anonyme et Odorannus révèle d'ailleurs, nous l'avons dit, la parenté des deux textes. Quelle est-elle exactement? Première hypothèse : Odorannus se serait inspiré de l'Historia Francorum Senonensis, ce qui est chronologiquement possible. Mais alors comment expliquer que la liste des bienfaits de Seguin soit beaucoup plus longue chez le premier, alors qu'elle se résume dans l'Historia, à la réfection de l'abbaye de Melun ? Il faudrait imaginer qu'Odorannus soit allé chercher le complément dans une deuxième source ce qui est possible mais inutilement compliqué.

\footnotetext{
33 Odorannus de Sens, Opera omnia, éd. Robert-Henri Bautier et Monique Gilles, Paris, Centre Nationale de la Recherche Scientifique, 1972, p. 84-113.

${ }^{34}$ Ibid., p. 96.

${ }^{35}$ Son récit de la trahison de Gautier commence en effet par «In ipso anno ».
} 
Il est plus simple, deuxième hypothèse, de postuler qu'Odorannus et l'anonyme de Sens soient allés chercher leurs informations sur Seguin dans une seule et unique source. L'anonyme se serait alors contenté d'en tirer ce qui l'intéressait, la réfection de Saint-Père de Melun, car il pouvait la relier à la trahison de Gautier, tandis qu'Odorannus recopiait l'intégralité des bienfaits de Seguin. Il est facile de comprendre pourquoi ce dernier faisait ce choix étant donné que l'un des buts de sa Chronique était de glorifier tous ceux qui avaient à un moment favorisé l'abbaye Saint-Pierre-le-Vif, à laquelle il appartenait. L'auteur de l'Historia Francorum Senonensis, au contraire, a donné une portée plus générale à son œuvre, et ne s'intéresse à l'action de Seguin que dans la mesure où il y trouve un lien avec l'événement qu'il raconte, ce qui est le cas ici.

Dès lors quelle peut être la source commune aux deux auteurs? C'est Odorannus qui nous donne l'indice le plus évident. La liste de bienfaits qu'il énumère est en effet très semblable dans la forme et dans l'esprit aux Gesta episcoporum que l'on peut trouver ici et là dans les diocèses à cette époque et dont l'exemple le plus proche de Sens sont les Gestes des évêques d'Auxerre. Si l'on postule que l'auteur de l'Historia Francorum Senonensis et Odorannus ont tour à tour puisé leurs informations dans une source de ce genre, peut-être d'ailleurs au même endroit, car rien n'interdit de penser que le premier était lui aussi moine à Saint-Pierre-le-Vif, on peut dès lors émettre l'existence de Gesta archiepiscoporum Senonensis dont certains historiens soupçonnaient déjà la possibilite ${ }^{36}$.

Notre auteur anonyme serait donc allé chercher la mention de la réfection de Saint-Père de Melun dans cet ouvrage en faisant l'erreur de

${ }^{36}$ Notamment Ferdinand Lot, «De la valeur historique de l'Historia Francorum Senonensis », appendice VII dans Les Derniers Carolingiens, Paris, Bibliothèque de l'École des hautes études, coll. «Bibliothèque de l'École des hautes études. Sciences philologiques et historiques », 1891, p. 338-345. 
la placer en 999 qui était en réalité la date de la mort de Seguin, indiquée au début de la notice le concernant.

On voit donc dans ce cas précis combien l'erreur d'un chroniqueur médiéval peut être parfois plus féconde à notre réflexion que sa rigoureuse infaillibilité. 